\title{
CARACTERIZAÇÃO DAS JANELAS DE OPORTUNIDADE NAS CONDIÇÕES DE TEMPO E MAR PARA OPERAÇÃO DAS FROTAS PESQUEIRAS INDUSTRIAIS DO SUL DO BRASIL
}

\author{
Manuela Luiza de Andrade Camisão', Patricia Sfair Sunye ${ }^{2}$ and Thiago Pereira Alves ${ }^{1}$
}

\begin{abstract}
Resumo
As frotas pesqueiras industriais atuantes no sul do Brasil possuem diferentes áreas de atuação, métodos de captura e espécies-alvo. No entanto, todas possuem limitações de operação de pesca e segurança associadas às condições de tempo e mar, e que estão associadas à eficiência econômica da atividade. Neste estudo, temos o objetivo de definir as condições de tempo e mar ideais para a operação de pesca de cada frota, caracterizando assim "janelas de oportunidade". As janelas serão caracterizadas e determinadas a partir de entrevistas semiestruturadas com mestres e armadores. Após a caracterização das janelas de captura será iniciada a etapa de validação. Nesta etapa, será investigado se as respectivas frotas operaram durante os dias em que as janelas de oportunidade para sua modalidade foram observadas. Como produto técnico e tecnológico, o projeto visa a disponibilização das informações, mediante boletins de previsão dessas janelas de oportunidade. A disponibilização desses boletins tem o intuito de contribuir na otimização do esforço de pesca dessas frotas e, consequentemente, no aumento da eficiência econômica das operações de pesca.
\end{abstract}

\section{Palavras-chave}

Pesca industrial, janelas de oportunidade, condições de tempo, mar

\section{Introdução}

A captura comercial de organismos aquáticos, no Brasil, é realizada através da pesca artesanal e industrial. A pesca industrial é a do tipo empresarial, realizada com embarcações motorizadas e de grande porte. Possui grande capacidade produtiva, atuação em áreas distantes da costa e autonomia de navegação (GIULIETTI ; ASSUMPÇÃO,1995). Os ambientes costeiros e oceânicos ao longo do litoral de Santa Catarina e Rio Grande do Sul possuem um dos maiores potenciais pesqueiros da costa brasileira (PEZZUTO; BENINCÁ, 2015). A ampla largura da plataforma associada ao aporte de nutrientes provenientes das descargas continentais e de correntes frias de origem subantártica são responsáveis pela abundância de recursos pesqueiros na região (Seeliger, 1998). A maior parte da pesca industrial brasileira está concentrada nesta região e opera, eventualmente, com algumas restrições batimétricas dependendo da licença específica, atuando sobre estoques demersais e pelágicos, com uma grande variedade de tipos de embarcações e petrechos de pesca (PEZZUTO; BENINCÁ, 2015).

Em Santa Catarina, Itajaí e Navegantes representam o maior pólo da pesca industrial no estado ( ; PETROBRAS, 2020) (Univali/Cttmar, 2003). Os municípios de Laguna e Porto Belo também são pontos importantes de desembarques da pesca industrial em Santa Catarina, porém menos representativos (PETROBRAS, 2020). Os recursos pesqueiros presentes em uma captura dependem da arte de pesca empregada e da vulnerabilidade das espécies aos petrechos utilizados (CASTELLO; KRUG, 2015). Dez tipologias de aparelho de pesca foram registradas nas descargas da frota industrial em Santa Catarina, dentre elas, as frotas de cerco, emalhe, arrasto de parelha, vara e isca-viva e arrasto duplo representaram, respectivamente, as maiores contribuições na produção do triênio 2017-2019. O cerco (traineiras) foi responsável por $44,4 \%$ da produção ( 87 mil toneladas) e o emalhe 15,2\% (30 mil toneladas). As modalidades de arrasto de parelha, vara e isca-viva e arrasto duplo vieram na sequência, representando cerca de $10 \%$ da produção industrial acumulada no período (PETROBRAS, 2020).

As descargas pela frota industrial, registradas em Santa Catarina, totalizaram 66.478,51 toneladas em 2017, $68.609,44$ toneladas em 2018 e $62.344,34$ toneladas em 2019. Juntos, os municípios de Itajaí e Navegantes

\footnotetext{
${ }^{1}$ Instituto Federal de Santa Catarina, Itajaí, Santa Catarina, Brasil ${ }^{2}$ Doutora em Oceanografia Biológica Patricia Sunye, Universidade do Estado de Santa Catarina (UDESC), Laguna, Santa Catarina, Itajaí, Santa Catarina

Emails: patricia.sunye@udesc.br (Patricia Sfair Sunye), thiago.alves@ifsc.edu.br (Thiago Pereira Alves)
}

\section{Corresponding author:}

Manuela Luiza de Andrade Camisão, Instituto Federal de Santa Catarina, Itajaí, Santa Catarina, Brasil

Email: manuelacamisao@gmail.com 
foram responsáveis por $95 \%$ da produção industrial de Santa Catarina, no período de 2017 a 2019, refletindo a grande concentração das descargas nesse polo pesqueiro (PETROBRAS, 2020). A sardinha é um dos principais recursos pesqueiros marinhos do Brasil, sua captura alimenta a indústria de enlatados, seja como matéria prima(enlatados de sardinha), ou de forma indireta, quando utilizada na captura do atum (bonito-listrado) pela frota de vara e iscaviva (CERGOLE ; DIAS-NETO, 2011). Em Santa Catarina, a sardinha foi a espécie mais capturada no triênio 2017-2019. A sardinha-verdadeira e a sardinha-lage, representaram, respectivamente, 33,5 e 31,6 mil toneladas acumuladas das descargas da pesca industrial no estado. Em terceiro e quarto lugar, a corvina e o bonito-listrado representaram, respectivamente, 29,8 e 21,5 mil toneladas.Juntas, essas quatro categorias responderam por cerca de 60\% das descargas da pesca industrial (PETROBRAS, 2020).

O clima interfere de muitas formas na atividade de pesca. Estudos sobre os efeitos do clima nos estoques pesqueiros brasileiros envolvem dois recursos principais, a sardinha Sardinella brasiliensis (SUNYE; SERVAIN, 1998, PAIVA; MOTTA 1999) e a tainha Mugil liza (VIEIRA; SCALABRINI, 1991). O impacto que uma condição meteorológica pode gerar depende de sua severidade e na sensibilidade de uma determinada atividade ou operação a essa condição. Na pesca, fenômenos meteorológicos podem tornar a navegação e as as atividades mais difíceis e perigosas (Wmo, 2018). Informações sobre mudanças na velocidade e direção do vento ao longo do dia para a área para qual estão navegando ou operando o vento são muito importantes para os pescadores. Os ventos podem exercer uma força considerável sobre as embarcações e petrechos e podem criar condições de trabalho perigosas. As ondas são geralmente o segundo elemento mais importante depois do vento. Ondas de vento têm efeitos significativos no avanço das embarcações, na rapidez com que os peixes podem ser encontrados e capturados e na produtividade das operações. Mudanças rápidas nas condições de ondulação representam riscos diretos para a integridade estrutural da embarcação, riscos para a estabilidade e operações de convés devido a despreparo da tripulação (Wmo, 2018).

No sul do Brasil, predomina o clima subtropical úmido, onde as massas de ar tropicais marítimas (MTM), úmidas e instáveis alcançam as costas e movem-se para seu interior carregando calor e umidade ao longo das frentes quentes e frias, onde o ar tropical encontra o ar polar. A precipitação é abundante durante todo o ano, mas atinge seus maiores valores no verão (MENDONÇA; DANNIOLIVEIRA, 2007). Para Ayoade (1996), sistemas produtores de tempo são sistemas de circulação acompanhados por padrões e tipos característicos de tempo. Eles causam variações diárias e semanais no tempo e são muitas vezes mencionados como sendo perturbações atmosféricas ou meteorológicas. O autor Machado (2019) definiu as frentes frias e os ciclones como sistemas meteorológicos causadores de tempestades e ventos fortes. Segundo o autor, esses sistemas interferem diretamente nas condições de agitação do mar.

Segundo Fulsas (2007), a atual previsão do tempo para a pesca é uma ferramenta muito importante para atividade e foi o resultado de um longo processo que envolveu meteorologistas e pescadores. Ainda segundo o autor, a principal diferença entre o conhecimento do tempo leigo e o profissional é a diferença na extensão das redes e, como consequência, a capacidade de manipular escala e distância. Para Ayoade (1996), o planejamento dos recursos climáticos envolve o uso racional dos efeitos benéficos do tempo e do clima e a prevenção, eliminação e minimização dos efeitos maléficos. Neste estudo, temos o objetivo de definir as condições de tempo e mar ideais para a operação de pesca denominada, "janelas de oportunidade", para cada frota pesqueira atuante na região sul do Brasil.

\section{Revisão bibliográfica}

Onde e quando ir pescar tem sido questionado desde que as pessoas começaram a pescar. A diferença nas capturas quando as decisões certas e erradas são feitas pode ser enorme (HART E REYNOLDS, 2004). Informações sobre o tempo podem contribuir para a redução de custos das atividades de pesca. Em 1999 a EPAGRI - Empresa de Pesquisa Agropecuária e Extensão Rural de Santa Catarina desenvolveu o Projeto METEOPESCA, que foi implantado em maio de 2000. O objetivo do projeto era aumentar a segurança dos pescadores (artesanais e industriais) durante as operações de pesca, para isso, foram formulados e boletins de previsão de tempo que foram repassados através de bases de radiocomunicação costeiras. Após o envio destas informações em forma de boletim, operadores de rádio coletavam informações de posição e condições de tempo com cerca de 15 embarcações situadas entre o Chuí e Paranaguá. Informações como esta, são valiosas para melhorar e aprimorar a previsão de tempo clima, em estudos sobre mudanças climáticas, na compreensão da interação entre o oceano e a atmosfera, na calibração de imagens de satélite, entre outros (LIMA; SUNYE; VIEIRA, 2009).

Segundo Coppini (2017) sem informações adequadas sobre as condições meteorológicas e oceanográficas, os pescadores possuem capacidade limitada de resposta e planejamento, o que pode ocasionar na perda de vidas, desastres ambientais e danos para a economia, sociedade e ecossistemas. Pescadores entrevistados pelo projeto METEOPESCA, relataram que a previsão implica numa redução de custos entre $10-30 \%$ por viagem. Ainda segundo a coleta de informações do projeto, a organização das viagens em função da previsão do tempo permite que os pescadores tomem decisões mais assertivas quanto ao local de captura, tempo de navegação, tipo de arte de pesca e espécie alvo (LIMA; SUNYE; VIEIRA, 2009) destacando a importância da previsão do tempo na tomada de decisão desses profissionais. A Epagri/Ciram ainda disponibiliza para a região sul (Chuí a Paranaguá), diariamente, a previsão para a navegação e pesca para 5 dias (EPAGRI/CIRAM, 2021). Também encontra-se disponível o "Boletim ao Mar", o novo aplicativo desenvolvido em parceria entre a Marinha do Brasil e o Instituto Rumo ao Mar - RUMAR (MARINHA DO BRASIL, c2021). Com relação aos boletins de tempo disponibilizados pelo METEOPESCA, os dados de superfície eram provenientes da rede de estações costeiras do estado de Santa Catarina e do Rio Grande do Sul ( ; LIMA; SUNYE; VIEIRA, 2009) (Sunye, Vieira, Pacheco, \& Kinceler, 2007), disponibilizando níveis 
de alerta (associados a diferentes cores) relacionados à velocidade do vento. O primeiro nível de alerta corresponde a ventos entre 40 e $50 \mathrm{~km} / \mathrm{h}$, que dificultam as operações de pesca; o segundo nível, ventos entre 50 e $60 \mathrm{~km} / \mathrm{h}$, que dificultam a navegação; o terceiro nível, entre 60 e 70km/h, indica as áreas a serem evitadas e o fechamento de portos e barras de acesso; e o quarto nível, ventos acima de $70 \mathrm{~km} / \mathrm{h}$, áreas com alerta máximo (LIMA; SUNYE; VIEIRA, 2009).

Embora a diversidade de artes de pesca seja interessante em si , mais surpreendente é a inovação contínua em forma e eficiência. As principais artes de pesca têm embarcações, petrechos e métodos de operação distintos. As redes de cerco capturam os cardumes de peixes cercandoos com uma enorme rede. As redes de arrasto filtram as massas de água em uma velocidade mais alta que a natação sustentável do peixe. Já a captura de espinhel atrai os peixes com as iscas e as redes de emalhar formam paredes de rede invisíveis nas quais os peixes nadam e ficam emaranhados (HART E REYNOLDS, 2004). No método de vara e isca-viva, a viagem de pesca pode ser dividida em duas etapas: uma dedicada à captura de isca-viva, e outra direcionada à captura das espéciesalvo (MADUREIRA; MONTEIRO-NETO, 2020). Apesar de utilizarem diferentes métodos de captura, todas as frotas estão suscetíveis às condições de tempo e mar. Por isso, a consciência situacional do mar (SSA) em termos da disseminação adequada de dados ambientais marinhos para usuários e partes interessadas é estrategicamente importante para a segurança e gestão financeira da atividade pesqueira de todas as diferentes frotas (LIMA; SUNYE; VIEIRA, 2009 ; ) (Coppini, 2017). O estabelecimento da confiança nas previsões meteorológicas parece ter sido um processo gradual, com três fatores cruciais: as previsões tornaramse acessíveis, eram considerados confiáveis e os usuários alimentaram expectativas realistas sobre como elas poderiam ser usadas (Fulsas, 2007). Pinardi et al. (2017), revisou em seu estudo, o desenvolvimento histórico de conceitos e práticas na ciência de previsão para os oceanos. Segundo os autores, as previsões permitem a verificação da "teoria" da atmosfera e do oceano por observação do mundo natural. Para o oceano, este passo fundamental no conhecimento não ocorreu até a década de 1980. A pesca é uma das atividades beneficiadas com previsões regulares para os oceanos.

Condições adversas de tempo podem, além de comprometer a segurança da tripulação e elevar custos, dificultar as operações de pesca (Coppini, 2017). Tupper (apud Yaakob e Chau, 2005), listou os motivos mais frequentes citados pelos pescadores da Nova Inglaterra para o encerramento das atividades de pesca. Segundo os pescadores, as artes perdem a capacidade de pescar, a embarcação não consegue permanecer engatada e a tripulação sofre com a exaustão ao tentar lutar contra o movimento da embarcação. Espera-se, que métodos de pesca distintos, sejam também afetados de maneira distinta pelas condições adversas de tempo e mar. $\mathrm{O}$ efeito dos movimentos do barco na eficiência das artes de pesca foi quantificado por Enerhauget et al (apud Yaakob e Chau, 2005), em seu trabalho, os autores demonstraram que os movimentos verticais da embarcação afetam o avanço das portas da rede de arrasto e produzem o movimento vertical das portas, fazendo com que elas percam o contato com o fundo. Isso causa efeitos adversos na eficiência da pesca de arrasto. Segundo Hart e Reynolds (2004) as artes de pesca passivas, como redes de emalhe são mais vulneráveis a danos causados pelo mau tempo devido ao longo período necessário para a pesca. Yaakob e Chau (2005) concluíram em seu estudo, que condições adversas (ondas e ventos) durante as monções na Península da Malásia foram responsáveis pela redução significativa de captura durante esses períodos. Conhecer e prever situações como estas é essencial para a gestão da atividade.

A janela de oportunidade (windows of opportunity) é conceituada como um curto período de tempo dentro do qual alguma ação pode ser realizada para atingir um resultado desejado. O conceito é utilizado em diversas áreas, política (REARDON;MARDEN, 2018) gestão empresarial (SHEPHERD; ZACHARAKIS, 2018), e até mesmo na medicina, Andersen (2003). Em alguns casos, é possível planejar e antecipar as janelas de oportunidade e, em seguida, agir de acordo quando a janela for aberta. $\mathrm{Na}$ pesca, condições de tempo e mar podem caracterizar "janelas de oportunidades" para a atividade e estão intimamente ligadas à eficiência econômica da atividade. Para Perez, Lucato, Andrade, \&amp; M Pezzuto, and Rodrigues-Ribeiro (1998) as capturas industriais descarregadas no porto de Itajaí são importantes e representativas para os estudos da dinâmica dos estoques em uma vasta parcela da plataforma continental do Sudeste e Sul. Além disso, a grande variedade de frotas que descarregam na região (PETROBRAS, 2020), fazem deste, o local ideal para estudos sobre o caracterização da frotas e do seu comportamento diante de diferentes condições de tempo e mar.

\section{Metodologia}

Para a caracterização das janelas de oportunidade (condições de tempo e mar ideais para pescar) serão realizadas entrevistas semiestruturadas com mestres e armadores representantes das cinco frotas (cerco, emalhe de fundo, arrasto, vara e isca-viva e espinhel) que descarregam no Porto de Itajaí. A entrevista semiestruturada possui maior flexibilidade (BOTELHO ; CRUZ, 2013), como nosso estudo aborda um tema pouco explorado, este tipo de entrevista possibilita adaptações e um aporte maior de informações. As entrevistas serão realizadas no momento do desembarque seguindo a metodologia estabelecida por Perez et al. (1998) e também utilizada por Petrobras (2020), com a adição de questionamentos relacionados à relação "operação de pesca/condições de tempo e mar".

A caracterização das frotas está sendo realizada através de revisão bibliográfica e poderá ser complementada com dados coletados através das entrevistas. Para a validação será efetuado um cadastro com uma parte representativa de embarcações de cada frota e mantido um canal de comunicação, mediante acordo com armador e mestres, para o acompanhamento de suas viagens durante um período determinado. A comunicação tem o intuito de identificar se foi possível a operação de pesca, ou não, durante as janelas caracterizadas pela etapa anterior. Os dados observados serão obtidos através de estações convencionais e automáticas da região costeira disponibilizados pelo INMET, documentos de Síntese Sinótica Mensal do CPTEC/INPE 
(mediante solicitação) e boletins de alerta pela MARINHA e EPAGRI/CIRAM.

\section{Resultados esperados}

Contribuir com a gestão da atividade pesqueira industrial a partir da caracterização das "janelas de oportunidade" para as cinco principais frotas da pesca industrial que descarregam no Porto de Itajaí. De forma mais específica, descrever as áreas de atuação, a operação de pesca das diferentes modalidades e caracterizar as condições meteorológicas e oceanográficas que formam as janelas de oportunidade para as frotas de arrasto, cerco, emalhe de fundo, espinhel e vara e isca-viva.

Como produto técnico e tecnológico, o projeto visa a disponibilização das informações, mediante boletins de previsão dessas janelas de oportunidade. A disponibilização desses boletins tem o intuito de contribuir na otimização do esforço de pesca dessas frotas e, consequentemente, no aumento da eficiência econômica das operações de pesca. Acreditamos também, que a determinação dessas janelas de captura auxilie na análise dos múltiplos fatores que interferem na dinâmica da produção pesqueira na região sul do Brasil.

\section{Referências}

Andersen, S. L. (2003). Trajectories of brain development: point of vulnerability or window of opportunity? Neuroscience \& Biobehavioral Reviews, 3-18.

Ayoade, J. O. (1996). Introdução à climatologia para os trópicos. Editora Bertrand, 382-382.

Botelho, J., Cruz, V. A. G., \& Metodologia. (n.d.). (Vol. 135). Pearson Prentice Hall, São Paulo.

Castello, J., \& Krug, L. C. (n.d.). Introdução à Ciências do Mar. Editora Textos, 602-2017.

Cergole, M. C., \& Dias-Neto, J. (2011). Plano de gestão para o uso sustentável da sardinha-verdadeira do brasil. Brasília, Ibama.

Coppini, G. (2017). SeaConditions: a web and mobile service for safer professional and recreational activities in the Mediterranean Sea. Nat. Hazards Earth Syst. Sci., v, 17, 533-547.

Epagri/Ciram. (n.d.). Retrieved from https://ciram .epagri.sc.gov.br/impressao/Controle ?command=ExibirPescaAcessoem: 15 deoutubrode 2021

Fulsas, N. (2007).

Giulietti, N., \& Assumpção, R. (1995). Indústria pesqueira no brasil. agricultura em são paulo. São Paulo.

Hart, P. J., Reynolds, J. D., \& Hart. (2004). Blackwell Publishing.

Lima, M., Sunye, P., \& Vieira, H. J. (2009). Projeto Meteopesca: a previsão do tempo como ferramenta para o aumento da segurança e produtividade da pesca em Santa Catarina. Boletim SBMET, 10-10.

Machado, J. (2019).

Madureira, L. S. P., \& Monteiro-Neto, C. (2020). Sustentabilidade da pesca do bonito-listrado no brasil. Rio de Janeiro.
Marinha, \& Brasil. (n.d.). Retrieved from https://www.marinha.mil.br/content/ aplicativo-boletim-ao-mar\#: : text $=$ Encontra $\% 2$ se $\% 20$ dispon $\%$ C $3 \%$ ADvel\%20para 20 download

Mendonça, F. D.-O., I, \& Climatologia, M. (2007).

Paiva, M. P., \& S, M. P. C. (1993). Capturas da sardinhaverdadeira. In Sardinella brasiliensis (Steindachner) (Osteichthyes: Clupeidae) e da fauna acompanhante (Vol. 32, p. 85-88).

Perez, J. A. A., Lucato, S. H. B., Andrade, A. H., \&amp; M Pezzuto, P. R., \& Rodrigues-Ribeiro. (1998).

Pezzuto, P., \& Benincà, E. M. (2015). Challenges in licensing the industrial double-rig trawl fisheries in Brazil. Latin American Journal of Aquatic Research $v, 43,496-513$.

Pinardi, N., Cavaleri, L., Coppini, G., Mey, P. D., Fratianni, C., Huthnance, J., \& Tibaldi, S. (2017). From weather to ocean predictions: an historical viewpoint. Journal of Marine Research(3), 103-159.

Projeto de Monitoramento da Atividade Pesqueira no Estado de Santa Catarina - PMAP-SC. Relatório Final - RTF BR 08042054/20. (n.d.). PETROBRAS.

Reardon, L., \& Marsden, G. (2018). Conclusion: A Window of Opportunity. Governance of the Smart Mobility Transition, 155-165.

Seeliger, U. (1998). Os ecossistemas costeiro e marinho do extremo sul do Brasil. Ecoscientia, 326-326.

Shepherd, D. A. ., \& Zacharakis, A. (2018).

Sunye, P., \& Servain, J. (1998).

Sunye, P., Vieira, H., Pacheco, R., \& Kinceler, L. M. (2007). A Rede de monitoramento costeiro do Projeto RECOS - Uso e apropriação de recursos costeiros - Institutos do Milênio. Boletim Paranaense de Geociências, 7788.

Univali/Cttmar. (2003). Boletim estatístico da pesca industrial de santa catarina - ano 2002. rel.téc.,univalicttmar. Itajaí.

Vieira, J., \& Scalabrin, C. (1991). (Vol. 13).

Wmo. (2018). Retrieved from https:// library.wmo.int/doc_num.php?explnum id $=5445$ Acessoem

Yaakob, O., \& Chau, Q. P. (2005). Weather downtime and its effect on fishing operation in Peninsular Malaysia. Jurnal Teknologi, 42, 13-26.

\section{Author Biographies}

Manuela Luiza de Andrade Camisão Camisão Mestranda do Programa de Pós-Graduação Stricto Sensu - Mestrado em Clima e Ambiente pelo Instituto Federal de Santa Catarina (IFSC)

Patricia Sfair Sunye Professora do Centro de Educação Superior da Região Sul (Ceres) Universidade do Estado de Santa Catarina (UDESC); Laguna, Santa Catarina;

Thiago Pereira Alves Doutor em Sistemas Costeiros e Oceânicos; Professor titular e orientador do Programa de Pós-Graduação Stricto Sensu - Mestrado em Clima e Ambiente; Instituto Federal de Santa Catarina (IFSC); Itajaí, Santa Catarina 